\section{Motorische Therapie bessert auch nicht motorische Symptome}

\author{
Treten Fatigue oder Depressionen bei Parkinson-Patienten vor allem in \\ Off-Phasen auf? Falls ja, gehen die nicht motorischen Symptome oft \\ deutlich zurück, wenn die motorische Therapie verbessert wird.
}

$\mathrm{M}$ orbus Parkinson betrifft nicht nur die Substantia nigra mit ihren dopaminergen Zellen, sondern auch viele andere Hirnareale, darunter den Locus coereolus mit noradrenergen Verbindungen oder den Nucleus basalis mit cholinergen Nervenzellen. Daran erinnerte Professor Heinz Reichmann, Universität Dresden. Es sei daher nicht erstaunlich, wenn Parkinson-Patienten typischerweise bis zu einem Dutzend nicht motorischer Beschwerden aufweisen, so Reichmann.

Deutlich häufiger als gleichaltrige $\mathrm{Pa}$ tienten ohne Parkinson kommt es bei den Betroffenen zu Geruchsstörungen, Gedächtnis- und Konzentrationsproblemen, Depressionen, Müdigkeit oder Interessenverlust. Reichmann erläuterte dies auch anhand von Daten der Priamo-Studie mit über 1.000 Patienten: Mehr als die Hälfte der Patienten berichteten über Fatigue und Ängste, jeweils 30-40\% nannten Schmerzen im Bein, Insomnie, Harndrang, Nocturie, Speichelfluss und Konzentrationsprobleme. Eine Therapie gegen die nicht motorischen Symptome scheint den Patienten oft ebenso wichtig wie die motorische Behandlung.

\section{Fatigue und Depression in Off-Phasen besonders häufig}

Für die Therapie sei es allerdings entscheidend, wann die Symptome auftreten, berichtete der Neurologe. Nach einer Studie werden nicht motorische Beschwerden wie Fatigue, Depressionen und Ängste bevorzugt während der Off-Phasen deutlich. In diesen Fällen sollten dann in erster Linie die motorische Therapie verbessert und die OffPhasen reduziert werden. Damit ließen oft auch nicht motorische Beschwerden nach, die an Off-Phasen gekoppelt sind. Ist keine Abhängigkeit von den Bewegungsphasen erkennbar, können sich Ärzte auf die symptomatische Therapie konzentrieren - bei einer Depression wären dann etwa SSRI oder NSRI indiziert.

Die Wahl der dopaminergen Arzneien hat jedoch ebenfalls einen Einfluss auf nicht motorische Symptome. Bei ausgeprägter Fatigue verwendet Reichmann Amantadin oder Rasagilin. Haben die Patienten Schlafstörungen, sind lang wirksame oder retardierte Dopaminagonisten von Vorteil. Wachen Patienten dennoch mitten in der Nacht auf und können nicht mehr einschlafen sind kurz wirksame Hypnotika eine Option. Bei exzessiver Tagesmüdigkeit kommt nach Auffassung des Parkinson-Experten eine Dosisreduktion der dopaminergen Arzneien infrage. Gegen die nicht ungefährlichen nächtlichen Bewegungen bei REM-Schlaf-Verhaltensstörungen nannte Reichmann Melatonin und Clonazepam.

\section{Antidementiva auch bei Parkinson-Demenz wirksam}

Ein weiteres Problem stellt die Parkinson-Demenz dar. Der kognitive Verfall und die Depression verschlechtern die Lebensqualität oft mehr als die Bewegungsstörung. Verbunden mit der Parkinson-Demenz sind zum einen AlphaSynuclein-Ablagerungen im Hirnstamm und Kortex, andererseits auch die Degeneration von Hirnkernen wie dem Nucleus basalis - einem der Hauptproduzenten von Acetylcholin. Deshalb können Cholinesterasehemmer wie das gegen Parkinson-Demenz zugelassene Rivastigmin von Vorteil sein, kürzlich wurde eine ähnlich gute Wirksamkeit auch für Donepezil gezeigt.

Thomas Müller

Symposium "Parkinson and Parkinsonism", 23. Meeting der European Neurological Society (ENS), Barcelona, 10.6.2013

\section{Bald Serummarker für Parkinson?}

Das pathologisch relevante Alpha-Synuclein bei Parkinson hat zu Lebzeiten der Patienten kaum eine diagnostische Bedeutung. Dies könnte sich in naher Zukunft ändern: Bei Parkinson-Patienten kommt es offenbar recht früh im Krankheitsverlauf zu einer verstärkten zytotoxischen Nitrosylierung von Alpha-Synuclein an ganz bestimmten Positionen. Möglicherweise ist die Synuclein-Nitrosylierung aber nicht nur ein Marker, sondern direkt an der Schädigung dopaminerger Neurone beteiligt. Die Arbeitsgruppe um Emilio Fernandez von der Universität in Sevilla hält nitrosyliertes Alpha-Synuclein-Protein sowohl für einen potenziellen Marker als auch für einen wichtigen Akteur bei der Parkinson-Pathogenese.

Thomas Müller

Poster-Session Nr 2, P594. Fernandez E et al. Cerebrospinal fluid and serum from Parkinso 23. Meeting der European Neurological Society (ENS), Barcelona, 8.-11.6.2013

\section{Parkinson-Frühdiagnose: Das Herz zeigt, was im Gehirn passiert}

Der eindeutige oder frühe Nachweis einer Parkinson-Erkrankung gestaltet sich schwierig. Die Dopamintransporter-Bildgebung per SPECT eignet sich nur bedingt. Zwar lassen sich damit andere Formen von Bewegungsstörungen, bei denen die Substantia nigra nicht betroffen ist, ausschließen, aber nicht eine Multisystematrophie. Dies ist jedoch mit der kardialen SPECT-Szintigrafie mit ${ }^{123}$ |-Metaiodobenzylguanidin (MIBG) möglich. Der radioaktive Tracer wird bevorzugt von sympathischen Nervenendigungen aufgenommen. Mit dem Verfahren lässt sich sowohl die Dichte der präsynaptischen sympathischen Nervenendigungen nachweisen als auch der präsynaptische funktionelle Tonus kardial-sympathischer Nerven, berichtete Professor Eduardo Tolosa, Barcelona. In einer Studie ließ sich eine Parkinson-Erkrankung mit einer Sensitivität von knapp 90\% und einer Spezifität von $83 \%$ von anderen neurodegenerativen Bewegungsstörungen (Multisystematrophie, progressive supranukleäre Blickparese, kortikobasale Degeneration) abgrenzen. Die ${ }^{123}$ |-MIBG-Szintigrafie kann daher ein nützliches - aber nicht eben kostengünstiges - Instrument zur Frühund Differenzialdiagnose sein, so Tolosa.

Thomas Müller

Symposium "Parkinson and Parkinsonism", 23. Meeting der European Neurological Society (ENS), Barcelona, 10.6.2013 